\title{
Relación entre estrategias de aprendizaje y rendimiento académico en alumnos de pedagogía en inglés
}

\author{
Juan-Carlos Cárdenas-Narváez
}

\section{RESUMEN}

El objetivo del estudio fue relacionar las estrategias de aprendizaje utilizadas por estudiantes de Pedagogía en Inglés de una universidad Chilena, representada por el puntaje alcanzado en el Cuestionario de Estrategias de Aprendizaje ACRA de Román et al. (1994, 2013), con su rendimiento académico. Se efectuó un estudio descriptivo correlacional. La muestra incluyó 60 estudiantes (45 mujeres y 15 varones), con rangos de edad entre 18 y 21 años. Los resultados indican un coeficiente correlacional aceptable. Se concluye que la escasa frecuencia de uso de algunas estrategias de aprendizaje comprueba la necesidad de reforzarlas sistemáticamente.

Palabras clave: estrategias de aprendizaje, rendimiento académico, aprendizaje de idiomas, ACRA, Chile. 


\section{Relação entre estratégias de aprendizagem e rendimento acadêmico em alunos de Pedagogia em Inglês}

\section{RESUMO}

O objetivo do estudo foi relacionar as estratégias de aprendizagem utilizadas por estudantes de Pedagogia em Inglês de uma universidade chilena, representada pela pontuação alcançada no Questionário de Estratégias de Aprendizagem ACRA (Aquisição, Codificação, Recuperação, Metacognitivas e Apoio) de Román et al. (1994, 2013), com seu rendimento acadêmico. Se efetuou um estudo descritivo de relação. A amostra incluiu 60 estudantes (45 mulheres e 15 homens), com idade entre 18 e 21 anos. Os resultados indicam um coeficiente de relação aceitável. Se conclui que a escassa frequência de uso de algumas estratégias de aprendizagem comprova a necessidade de reforçá-las sistematicamente.

Palavras chave: estratégias de aprendizagem, rendimento acadêmico, aprendizagem de idiomas, Chile.

\section{Relationship between learning strategies and academic performance in students of English Pedagogy}

\section{ABSTRACT}

The objective of the study was to relate the learning strategies used by students of English Pedagogy at a Chilean university, represented by the score reached in the ACRA Learning Strategies Questionnaire (Acquisition, Codification, Recovery, Metacognitives and Support) of Román et al. (1994, 2013) with their academic performance. A descriptive correlational study was carried out. The sample included 60 students (45 women and 15 men), with age ranges between 18 and 21 years. The results indicate an acceptable correlation coefficient. It is concluded that the low frequency of use of some learning strategies proves the need to systematically reinforce them.

Key words: learning strategies, academic performance, language learning, Chile. 


\section{Introducción}

El aprendizaje es un proceso personal, por lo cual las características propias de cada sujeto inciden en la forma en que se genera; a lo anterior se suma el contexto, el tipo de contenidos y otros aspectos de naturaleza socio-afectiva, como las expectativas, motivaciones y autopercepción. Reconociendo el carácter multidimensional y complejo del mismo, se espera profundizar en una de estas variables, a saber, las estrategias de aprendizaje.

El objetivo de esta investigación es identificar las estrategias de aprendizaje utilizadas por los alumnos de la carrera de Pedagogía en Inglés de una universidad de la zona central de Chile, y explicar el grado de correlación entre la frecuencia de uso de dichas estrategias con los resultados académicos de los estudiantes. La hipótesis de investigación establece la posible existencia de una correlación significativa entre las estrategias de aprendizaje utilizadas por los alumnos y el rendimiento académico, expresado en sus calificaciones.

Para dar respuesta a los objetivos, se optó por la metodología cuantitativa a través de un diseño descriptivo, correlacional-no experimental. La muestra fue seleccionada bajo el criterio de disponibilidad e incluyó a 60 alumnos ingresados a la universidad a comienzos del 2015. A fines de ese año, se aplicó el Cuestionario de Estrategias de Aprendizaje ACRA (Adquisición, Codificación, Recuperación, Metacognitivas y Apoyo) a los sujetos que componen la muestra, cuyos resultados fueron analizados y correlacionados con el rendimiento académico expresado en las calificaciones obtenidas por éstos durante el primer año de estudios en la universidad. Para establecer la confiabilidad del instrumento aplicado se utilizó el Alfa de Cronbach, y la validez del constructo se determinó mediante análisis factorial. Del mismo modo, se utilizó el Coeficiente de Pearson para los análisis correlacionales entre variables.

\section{Planteamiento del problema}

Actualmente, los estudios realizados en el ámbito de la educación, sobre todo los de la línea de la psicología cognitiva, han puesto especial énfasis en los procesos cognitivos que genera el aprendizaje. En la misma línea, el proceso de Reforma Educacional implementado en Chile a mediados de la década de 1990, puso énfasis en el desarrollo de habilidades, transformando el contenido en un medio para lograr el desarrollo de las mismas, más que en un fin en sí mismo. Este acento responde, sin lugar a dudas, a las características de la sociedad de hoy en día; sociedad en la cual el conocimiento se acrecienta diariamente debido a la expansión y rapidez de los cambios, por tanto, es una fuente inagotable y en constante revisión, imposible de abordar en forma completa.

En este contexto, se espera que en la práctica educativa de la enseñanza del inglés como lengua extranjera, el docente actúe como un mediador, apoyando al alumno a desarrollar aprendizajes efectivos a través de actividades y medios adecuados.

Sin embargo, a pesar de la puesta en marcha de la Reforma Educacional chilena, aún se mantienen las prácticas tradicionales, y poco se hace en cuanto a lograr en los alumnos el desarrollo de habilidades. Por el contrario, el docente sigue siendo una fuente de información, y el alumno un mero receptor. En este sentido, el concepto de estrategias de aprendizaje y desarrollo de la metacognición, son aspectos poco abordados en el aula como parte del quehacer cotidiano, no obstante revierten una especial importancia, ya que al parecer inciden en el aprendizaje de los alumnos, y por tanto, en los resultados académicos.

En el sistema educativo actual se da especial importancia a los resultados académicos obtenidos por los alumnos durante su enseñanza media, ya que éstos son considerados en los procesos de selección para ingresar a la enseñanza universitaria.

En cuanto a los bajos resultados académicos alcanzados por los alumnos de enseñanza media en 
Chile, éstos son atribuidos principalmente a la falta de estudio; no obstante, poco se consideran - en la práctica educativa - otros aspectos relacionados con el rendimiento, como es la motivación y las características personales. Entre éstas, un aspecto muy importante está constituido por las estrategias de aprendizaje que debieran utilizar los estudiantes cuando enfrentan un desafío académico. Incluso es probable que los alumnos no conozcan estas estrategias y, por tanto, no sean capaces de darles un uso adecuado; esta situación, bien manejada tanto por los alumnos como por los docentes, podría incidir favorablemente en sus resultados académicos. Muchos de los estudiantes desconocen las estrategias; algunos las usan de manera intuitiva, ya que así las adquirieron de otros, y son escasos los que las aplican en forma consciente (Beltrán, 1993).

De igual forma, los planes y programas específicos de la asignatura de inglés del sistema educativo chileno, enfatizan la adquisición de técnicas de lectura durante la etapa de enseñanza básica, para ser luego utilizadas durante la enseñanza media, "donde se destaca la comprensión lectora, aspecto que reconoce la funcionalidad de la lectura como medio de aprendizaje en una cultura letrada como la nuestra" (Vidal-Abarca, 1991).

Finalmente, se critica la insuficiente formación que traen los estudiantes al momento de ingresar a la educación universitaria, debido a que los estudios de nivel superior requieren del alumno mayores habilidades que las que poseía en la enseñanza media, tales como la capacidad para desarrollar aprendizajes autónomos mientras lee, realizar escritos, escuchar y participar presentando sus ideas en clases (Beltrán, 1993).

\section{Antecedentes teóricos}

El estudio de las estrategias de aprendizaje y uso de segundas lenguas data de los años ochenta, pudiendo distinguirse tres subcampos que corresponderían a teoría, investigación y enseñanza. En el contexto de la teoría, se cuenta con varios estudios cuya publicación data principalmente de los años noventa. Estos trabajos se centraron en cuestiones de definición y clasificación (Chamot et al., 1994a; Hsiao et al., 2002: 303-318; McDonough, 1995; O’Malley et al., 1990; Oxford et al., 1992: 1-35).

Por otra parte, varios investigadores (Dornyei $e t$ al., 2003; Manchón, 1998: 101-118, 2001: 27-70); McDonough, 1995) han señalado la falta de un verdadero y serio debate teórico en este campo, y las consecuencias que este hecho tiene a la hora de valorar la fundamentación y hallazgos de la investigación empírica llevada a cabo.

En cuanto a la investigación empírica, pueden distinguirse dos tipos de trabajos: estudios descriptivos sobre el uso de estrategias y los factores que condicionan dicho uso (McDonough, 1995, ofrece una revisión bastante completa), y trabajos empíricos sobre implementación de programas de entrenamiento estratégico.

A la luz de estos trabajos y concretamente en lo que respecta al modo en que se ha definido operacionalmente el constructo "estrategia", se observa la necesidad de revisar las estrategias objeto de análisis en cada trabajo, con el fin discernir con claridad la verdadera naturaleza de la relación entre uso de estrategias y avance en el aprendizaje. En este sentido, tal como afirma McDonough (1995) en su revisión sobre este tema, es esencial poder dilucidar si las estrategias contribuyen al aprendizaje ("an aid to learning") o son el resultado del aprendizaje ("the result of the learning").

Respecto a planteamientos pedagógicos acerca del proceso de enseñanza, existen propuestas bien conocidas sobre las razones que aconsejan la introducción de programas de entrenamiento estratégico en la enseñanza de segundas lenguas, así como el procedimiento que debería seguirse en la implementación de dichos programas (Wenden, 1991). De ello se ha derivado también un conjunto de materiales didácticos tanto para profesores como para alumnos (Ellis et al., 1989; Mendelsohn, 1994: 132-149; 
Rubin et al., 1994: 120; Weaber et al., 1997; Wenden, 1991; Willing, 1989). No obstante, con la excepción de dos trabajos que arrojan cierta luz sobre el tema (Rees-Miller, 1990: 776-781; Ridgway, 2000), no se conocen estudios que hayan analizado exhaustiva y críticamente estas propuestas a la vista de, primero, las teorías y modelos explicativos del aprendizaje de segundas lenguas aparecidas en la última década y, segundo, los planteamientos de la enseñanza procesual ("process teaching") en psicología de la educación.

En cuanto al contexto norteamericano y europeo, existen tres importantes grupos de investigación con base en Estados Unidos que han trabajado monográficamente sobre el tema: los equipos de la Universidad de Georgetown, liderados por la investigadora Ana Chamot; los trabajos dirigidos por Rebecca Oxford y, finalmente, el grupo de la Universidad de Minnesota, cuyo investigador principal es Andrew Cohen.

A excepción del grupo de Oxford, que ha llevado a cabo varios trabajos empíricos y realizado algunas incursiones en el análisis de aspectos metodológicos de la investigación existente, el foco de la investigación de estos grupos ha sido el diseño y/o análisis de programas de entrenamiento estratégico, tanto para estudiantes de segundas lenguas o bien para profesores encargados de llevar a cabo instrucción en estrategias.

Desde la perspectiva de la investigación empírica, debe destacarse también el trabajo en estrategias de vocabulario llevado acabo en la Universidad de Nottingham, Inglaterra, así como la investigación realizada por el grupo de la Universidad de Barcelona liderado por Carmen Muñoz, sobre la influencia del factor edad en el uso de estrategias de aprendizajes en aprendices de inglés como lengua extranjera. De igual forma, Manchón et al. (1997) han contribuido a nuestro conocimiento acerca de la influencia de tres variables independientes: nivel de dominio de la segunda lengua, tipo de texto escrito (narración/argumentación) y lengua usada en la escritura (lengua materna/extranjera), sobre los procesos y estrategias de escritura.
En términos generales, los distintos estudios en los que se integra la investigación empírica mencionada, no han tenido como meta el análisis exhaustivo de las bases teóricas y metodológicas de la investigación precedente, ni en términos globales ni en lo que respecta al grupo concreto de estrategias objeto de estudio por los distintos grupos de investigación. Como excepción, pueden mencionarse algunos trabajos sobre las estrategias de escritura, como el realizado por Manchón (2001: 27-70).

\section{Método Objetivo general}

En la necesidad de identificar las estrategias de aprendizaje que los estudiantes tienen al momento de ingresar a la educación universitaria, esta investigación se propuso: "determinar el grado de correlación entre la frecuencia de uso de estrategias de aprendizaje utilizadas por alumnos de Pedagogía en Inglés de una universidad de la zona central de Chile, representado por el puntaje alcanzado en el Cuestionario ACRA, y el rendimiento académico obtenido durante el primer año del programa".

\section{Objetivos específicos}

Esta investigación se planteó los siguientes objetivos específicos:

1. Identificar la frecuencia de uso de las estrategias de aprendizaje, según Modelo ACRA, por parte de los sujetos de la muestra.

2. Establecer el grado de asociación (correlación) entre la frecuencia de uso de las estrategias de aprendizaje ACRA y las calificaciones obtenidas por la muestra al final del primer año de la carrera.

\section{Hipótesis de trabajo}

Debido a la importancia que diversos trabajos de investigación asignan a las estrategias de aprendizaje como factor determinante en el rendimiento académico; la necesidad de conocer en qué medida los alumnos de la muestra hacen uso de ellas, y para determinar si 
esta frecuencia de uso explica el rendimiento académico de los sujetos de estudio, la presente investigación se planteó la siguiente hipótesis de trabajo:

- Existe correlación significativa $(a=5 \%)$ entre los puntajes alcanzados por los estudiantes en las escalas acra y su rendimiento académico en la asignatura Competencias Comunicativas Integradas en Inglés II.

\section{Muestra y tipo de estudio}

Se efectuó un estudio descriptivo correlacional. La muestra incluyó 60 estudiantes (45 mujeres y 25 varones) de segundo año de la carrera de Pedagogía en Inglés de una universidad de la zona central de Chile. La muestra fue seleccionada de forma no probabilística. El rango de edad de los participantes osciló entre los 18 y los 21 años. Todos ellos provienen de condición socioeconómica media, acorde con los datos aportados por la institución. Previo a la aplicación del Cuestionario ACRA, los sujetos de la muestra fueron informados acerca del estudio y firmaron una carta de consentimiento informado, de acuerdo con las disposiciones éticas de la institución.

\section{Diseño de la investigación}

Se trató de un estudio de corte no-experimental, ya que no se manipuló la variable rendimiento académico sino que se observó su comportamiento; con carácter descriptivo, ya que definió las frecuencias de uso de las diversas estrategias de aprendizaje; y correlacional, puesto que analizó el posible grado de asociación (correlación) entre los puntajes alcanzados por los alumnos en el Cuestionario ACRA y su rendimiento académico.

\section{Descripción del instrumento de recolección de datos}

Para la recolección de datos relacionados con la frecuencia de uso de estrategias de aprendizaje, se utilizaron las escalas ACRA (Román et al., 1994; revisión de Román et al., 2013). El instrumento consta de un cuestionario de 119 preguntas divididas en cinco escalas independientes que evalúan el uso que habitualmente hacen los estudiantes de: siete estrategias de adquisición de la información (I); 12 estrategias de codificación de la información (II); cuatro estrategias de recuperación de la información (III); tres estrategias metacognitivas (IV), y seis estrategias de apoyo al procesamiento o socioafectivas (V). Estas escalas pueden ser aplicadas en distintas fases del proceso educativo y con diferentes objetivos (evaluación inicial, de seguimiento o final), ya sea mediante procesos de intervención psicoeducativa:

a) preventiva (entrenar en determinada estrategia cognitiva de aprendizaje antes de que se prevea su uso)

b) correctiva (entrenar en determinada estrategia general tras constatar que su carencia o su incorrecta utilización afecta significativamente el rendimiento de los alumnos)

c) optimizadora (entrenar en determinada estrategia a un alumno o a un grupo de alumnos, quienes aunque usen algunas estrategias, puede que lo hagan en forma intuitiva, y por lo tanto, es adecuado automatizar su utilización).

Además, este instrumento de autoinforme, inspirado en los principios cognitivos de procesamiento de la información, permite evaluar de forma cuantitativa y cualitativa diversas estrategias cognitivas generales que utilizan los estudiantes en el aprendizaje que tiene lugar durante la actividad de estudio en sus distintas fases. Consta de un cuestionario que se conforma de: 20 preguntas para la escala de Adquisición; 46 para Codificación; 18 para Recuperación; 17 para Metacognitivas y 18 preguntas de la escala de Apoyo; además de una hoja de respuesta.

La validez de contenido fue calculada mediante el "método de juicio de expertos" y fue la siguiente: $r=0.87$ para la escala I, $r=0.89$ para la escala II, 
$r=0.91$ para la escala III, $r=0.88$ para la escala IV y $r=0.88$ para la escala V. La validez de constructo, calculada mediante el "método de juicio de expertos", fue de: $r=0.87$ para la escala $\mathrm{I}, r=0.87$ para la escala II, $r=0.89$ para la escala III, $r=0.88$ para la escala IV y $r=0.88$ para la escala V. De igual forma, la validez de constructo calculada mediante el "método de grado de acuerdo", y que proporciona valores análogos a los de una correlación, arrojó los siguientes valores: $r=0.9062$ para la escala $\mathrm{I} ; r=$ 0.8664 para la escala II; $r=0.9746$ para la escala III; $r=0.9591$ para la escala IV y $r=0.9591$ para la escala V. Respecto a la fiabilidad del instrumento, los resultados por escala son mostrados en la tabla 1; en la tabla 2 se ilustran las estrategias que componen cada escala y los ítems que las definen operacionalmente.

Tabla 1. Indicadores de fiabilidad de las Escalas ACRA

\begin{tabular}{|l|c|c|c|}
\hline \multicolumn{1}{|c|}{ Escalas } & $\begin{array}{c}\text { Alfa de Cronbach } \\
\text { estandarizada }\end{array}$ & $\begin{array}{c}\text { Pares/ impares Spearman- } \\
\text { Brown }\end{array}$ & $\begin{array}{c}\text { Mitades aleatorias* } \\
\text { Guttman }\end{array}$ \\
\hline Adquisición & 0.7144 & 0.6130 & 0.6130 \\
\hline Codificación & 0.9075 & 0.7955 & 0.7955 \\
\hline Recuperación & 0.8384 & 0.7160 & 0.7160 \\
\hline Metacognitivas & 0.8990 & 0.7337 & 0.7338 \\
\hline Apoyo & 0.8990 & 0.7337 & 0.7338 \\
\hline
\end{tabular}

*Corrección de Spearman-Brown.

Fuente: Elaboración propia.

Tabla 2. Resumen Escalas ACRA

\begin{tabular}{|c|c|c|}
\hline \multicolumn{3}{|c|}{ I. Escala de estrategias de adquisición de información } \\
\hline Núm. estrategia & Ítems que la definen operativamente estandarizada & Total: 20 \\
\hline 1. Exploración & $1-3-11$ & (3) \\
\hline 2. Subrayado lineal & $5-8$ & $(2)$ \\
\hline 3. Subrayado idiosincrático & $6-7-10$ & (3) \\
\hline 4. Epigrafiado & $2-9$ & $(2)$ \\
\hline 5. Repaso en voz alta & $13-14-16-19$ & (4) \\
\hline 6. Repaso mental & $4-15-17-18$ & (4) \\
\hline 7. Repaso reiterado & $12-20$ & $(2)$ \\
\hline \multicolumn{3}{|c|}{ II. Escala de estrategias de codificación de información } \\
\hline Núm. estrategia & Ítems que la definen operativamente estandarizada & Total: 26 \\
\hline 8. Nemotecnias & $43-44-45-46$ & (4) \\
\hline 9. Relaciones intracontenido & $3-4-5-29$ & (4) \\
\hline 10. Relaciones compartidas & $8-9-10$ & (3) \\
\hline
\end{tabular}




\begin{tabular}{|c|c|c|}
\hline 11. Imágenes & $11-12-13$ & (3) \\
\hline 12. Metáforas & $14-15$ & (2) \\
\hline 13. Aplicaciones & $6-7-16-17-18-19$ & (6) \\
\hline 14. Autopreguntas & $21-22-23-27-28$ & (5) \\
\hline 15. Paráfrasis & $20-24-25-26$ & (4) \\
\hline 16. Agrupamientos & $30-31-32-33-34-42$ & (6) \\
\hline 17. Secuencias & $35-36$ & (2) \\
\hline 18. Mapas conceptuales & $38-39$ & (2) \\
\hline 19. Diagramas & $1-2-37-40-41$ & (5) \\
\hline \multicolumn{3}{|c|}{ III-Escala de Estrategias de Recuperación de Información } \\
\hline Núm. estrategia & Ítems que la definen operativamente estandarizada & Total: 18 \\
\hline 20. Búsqueda de codificaciones & $1-2-3-4-10$ & (5) \\
\hline 21. Búsqueda de indicios & $5-6-7-8-9$ & (5) \\
\hline 22. Planificación de respuesta & $11-12-14-17-18$ & (5) \\
\hline 23. Respuesta escrita & $13-15-16$ & (3) \\
\hline \multicolumn{3}{|c|}{ IIV. Escala de estrategias metacognitivas } \\
\hline Núm. estrategia & Ítems que la definen operativamente estandarizada & Total: 17 \\
\hline 24. Autoconocimiento & $1-2-3-4-5-6-7$ & (7) \\
\hline 25. Automanejo/planificación & $10-11-12-13$ & (4) \\
\hline 26. Automanejo/regulación y evaluación & $8-9-14-15-16-17$ & (6) \\
\hline \multicolumn{3}{|c|}{ IIV. Escala de estrategias metacognitivas } \\
\hline Núm. estrategia & Ítems que la definen operativamente estandarizada & Total: 18 \\
\hline 27. Autoinstrucciones & $1-3-4-13$ & (4) \\
\hline 28. Autocontrol & 2 & (1) \\
\hline 29. Contradistractoras & $5-6-7$ & (3) \\
\hline 30. Interacciones sociales & 8-9-10-11-12 & (5) \\
\hline 31. Motivación intrínseca y extrínseca & $14-15-16-17$ & (4) \\
\hline 32. Motivación de escape & 18 & (1) \\
\hline
\end{tabular}

Fuente: Román et al. 1994; revisión de Román y Poggioli 2013. 


\section{Procedimiento}

Se invitó a los estudiantes que conformaron la muestra a participar del estudio mediante una carta, en la cual se informó de los objetivos de la investigación, así como los aspectos éticos, esto es, el manejo discreto de la información entregada por ellos. Finalmente, el grupo firmó un formato de consentimiento informado y se aplicó el cuestionario ACRA un día de semana en horario matutino. Una vez colectadas las hojas de respuesta, se procedió a su tabulación, asignando en cada escala el valor 1 a la respuesta $\mathrm{A}$ (nunca o casi nunca); 2 a la respuesta $\mathrm{B}$ (algunas veces); 3 a la $\mathrm{C}$ (bastantes veces) y 4 a la respuesta $\mathrm{D}$ (siempre o casi siempre). Dentro de cada escala, los puntajes A fueron multiplicados por 1; los puntajes B por 2; los puntajes $\mathrm{C}$ por tres y los D por 4, lo cual constituyó la puntuación directa total. El total de protocolos válidos fue de 60 hojas de respuesta contestadas. Se tabularon los resultados y fueron analizados estadísticamente mediante el software SPSS 18 (Statiscal Package for the Social Sciences).

Para la variable Rendimiento Académico, en la asignatura de Competencias Comunicativas Integradas en Inglés II: Vida Cotidiana, se utilizó la técnica de registro de notas, en la cual se identificó la nota obtenida por cada miembro de la muestra. Estos datos fueron solicitados formalmente a la Dirección de Escuela de la carrera en cuestión. Para efectos del estudio se incluyó la nota promedio obtenida al final del curso en la habilidad de "Reading", toda vez que las estrategias
ACRA apoyan el proceso de lectura comprensiva. En cuanto al análisis de datos, en relación con el objetivo específico 1, se realizó un análisis global del porcentaje de uso que los estudiantes declaran hacer de las 32 estrategias de aprendizaje ACRA, tomando como referencia que el rango de frecuencia de utilización sería determinado por el porcentaje de uso que los alumnos declara hacer de la estrategia respectiva. De esta forma, se procedió a realizar los siguientes pasos: para detectar la frecuencia de uso de cada uno de los grupos de estrategias (Adquisición, Codificación, Recuperación, Metacognitivas y Apoyo) por separado, se procedió a contar las A, B, C y D tabuladas a partir de las respuestas emitidas por los estudiantes. Luego, para representar analíticamente la frecuencia de uso de las diferentes estrategias de aprendizaje que los estudiantes declararon hacer, se confeccionó una serie de tablas que presentan la frecuencia de uso clasificada en cuatro niveles: "Muy frecuentemente" (mf), equivalente al grado D de las escalas ACRA (siempre o casi siempre); "Frecuentemente" (f), equivalente al grado C (bastantes veces); "Medianamente frecuente" (mdf), equivalente al grado B (algunas veces) y "Baja frecuencia" (bf), equivalente al grado A de las ACRA (nunca o casi nunca). Posteriormente, a cada uno de los niveles se asignó un rango de frecuencia acorde al porcentaje de uso, y se establecieron cuatro niveles de significancia en relación con la frecuencia de uso antes mencionada. La tabla 3 ilustra estos rangos.

Tabla 3. Rango de frecuencia y nivel de significancia, de acuerdo con el nivel de uso, de las diferentes estrategias ACRA

\begin{tabular}{|l|l|l|l|}
\hline & \multicolumn{1}{|c|}{ Equivalencia ACRA } & Rango de frecuencia & \multicolumn{1}{c|}{ Nivel de significancia } \\
\hline Muy frecuentemente (mf) & Grado D (Siempre/casi siempre) & Sobre un 75\% & Alta significancia \\
\hline Frecuentemente (f) & Grado C (Bastantes veces) & Entre un 74\% y un 50\% & Significante \\
\hline Medianamente frecuente (mdf) & Grado B (Algunas veces) & Entre un 49\% y un 25\% & Medianamente significante \\
\hline Baja frecuencia (bf) & Grado A (Nunca o casi nunca) & Menos del 24\% & Baja significancia \\
\hline
\end{tabular}

Fuente: Elaboración propia. 


\section{Resultados}

\section{Frecuencia de uso de las estrategias de aprendizaje}

Los datos de frecuencia de uso de estrategias en cada una de las cinco escalas ACRA son presentados en las siguientes tablas.

De acuerdo con estos datos, en la tabla 4 se observa que las estrategias de carácter atencional, como la "Exploración", "Subrayado lineal", "Subrayado idiosincrático" y "Epigrafiado", se ubicaron en el rango "Medianamente frecuente", esto es, sólo entre un 25 y $49 \%$ de frecuencia de uso, por lo tanto se consideran "Medianamente significativas". Del mismo modo, las estrategias de fragmentación, como el "Repaso en voz alta" y "Repaso mental", se manifestaron en rango "Medianamente Frecuente" (25-49\%), lo cual las hace medianamente significativas. Luego, la estrategia descrita con uso "Frecuentemente" (50-74\%) corresponde al "Repaso reiterado", es decir, con una frecuencia de uso "Significativa".
Según la tabla 5, de las 12 estrategias incluidas en la escala de Codificación, las cuales se clasifican como procesos de Nemotecnización, Elaboración y Organización, las estrategias de "Nemotecnias", "Relaciones intra-contenido", "Relaciones compartidas", "Imágenes", "Diagramas", "Auto-preguntas", "Paráfrasis" y "Agrupamientos", se presentaron en un rango de frecuencia de uso de $25 \%$ a $49 \%$ (mdf), lo que implica "mediana significatividad". Por otra parte, las Estrategias de Elaboración, como "Metáforas" y "Aplicaciones", alcanzan nivel "Significante", con un rango de uso de $50 \%$ a $74 \%$ (f). De igual forma, la estrategia "Secuencias" alcanza el nivel "Frecuentemente", con un rango de $50 \%$ a $74 \%$, es decir, esta estrategia es también significativa. Sin embargo, la mayor diferencia en el rango de frecuencia de uso se produce con la estrategia "Mapas conceptuales", la cual se presenta en un nivel inferior al 25\% (bf), con una significación mínima. Este resultado es interesante ya que el uso de esta estrategia es bastante difundido en los diferentes niveles educacionales en Chile.

Tabla 4. Frecuencia de uso estrategias.

Escala Adquisición de información

\begin{tabular}{|l|l|c|}
\hline \multicolumn{1}{|c|}{$\mathbf{N}^{\mathbf{1}}$} & \multicolumn{1}{|c|}{ Estrategia } & Frecuencia \\
\hline 1 & Exploración & mdf \\
\hline 2 & Subrayado lineal & $\mathrm{mdf}$ \\
\hline 3 & Subrayado idiosincrático & $\mathrm{mdf}$ \\
\hline 4 & Epigrafiado & $\mathrm{mdf}$ \\
\hline 5 & Repaso en voz alta & $\mathrm{mdf}$ \\
\hline 6 & Repaso mental & $\mathrm{mdf}$ \\
\hline 7 & Repaso reiterado & $\mathrm{f}$ \\
\hline & Resumen: & 0 \\
\hline & Estrategias usadas mf & 1 \\
\hline & Estrategias usadas $\mathrm{f}$ & 6 \\
\hline & Estrategias usadas $\mathrm{mfd}$ & 0 \\
\hline & Estrategias usadas bf & 7 \\
\hline & Total estrategias & \\
\hline
\end{tabular}

Fuente: Elaboración propia. 
Tabla 5. Frecuencia de uso estrategias. Escala de Codificación

\begin{tabular}{|c|c|c|}
\hline$N^{\circ}$ & Estrategia & Frecuencia \\
\hline 8 & Nemotecnias & mdf \\
\hline 9 & Relaciones Intracontenido & mdf \\
\hline 10 & Relaciones compartidas & mdf \\
\hline 11 & Imágenes & mdf \\
\hline 12 & Metáforas & $f$ \\
\hline 13 & Aplicaciones & $f$ \\
\hline 14 & Autopreguntas & mdf \\
\hline 15 & Paráfrasis & mdf \\
\hline 16 & Agrupamientos & mdf \\
\hline 17 & Secuencias & $f$ \\
\hline 18 & Mapas conceptuales & bf \\
\hline \multirow[t]{7}{*}{19} & Diagramas & mdf \\
\hline & Resumen: & \\
\hline & Estrategias usadas mf & 0 \\
\hline & Estrategias usadas $\mathrm{f}$ & 3 \\
\hline & Estrategias usadas mfd & 8 \\
\hline & Estrategias usadas bf & 1 \\
\hline & Total estrategias & 12 \\
\hline
\end{tabular}

Fuente: Elaboración propia.

En cuanto a la escala de Recuperación o Evocación, la cual determina en qué medida los sujetos de la muestra utilizan estrategias que favorecen la búsqueda de información en la memoria y la generación eventual de respuesta, se determinó que las cuatro estrategias que la componen: "Búsqueda de codificaciones", "Búsqueda de indicios", "Planificación de respuesta" y "Respuesta escrita", se presentaron con una frecuencia ubicada en el rango de $50 \%$ a $74 \%$ (f), es decir, su frecuencia de utilización es significativa.
Respecto a la escala de Estrategias Metacognitivas (tabla 7), las cuales suponen y apoyan, por una parte, el conocimiento que un individuo tiene acerca de los propios procesos en general, y de estrategias cognitivas de aprendizaje en particular, y por otra, la capacidad de manejo de las mismas, las tres estrategias que la componen, "Autoconocimiento", "Automanejo/planificación", y "Automanejo/regulación y evaluación", los resultados obtenidos indican que estas estrategias son significativas en cuanto a su frecuencia de uso, ya que se ubicaron en un rango de utilización del $50 \%$ a $74 \%$ (f). 
Tabla 6. Frecuencia de uso estrategias. Escala de Recuperación

\begin{tabular}{|l|l|c|}
\hline \multicolumn{1}{|c|}{$\mathbf{N}^{\mathbf{1}}$} & \multicolumn{1}{|c|}{ Estrategia } & Frecuencia \\
\hline 20 & Búsqueda de codificaciones & $\mathrm{f}$ \\
\hline 21 & Búsqueda de indicios & $\mathrm{f}$ \\
\hline 22 & Planificación de respuestas & $\mathrm{f}$ \\
\hline 23 & Respuesta escrita & $\mathrm{f}$ \\
\hline & Resumen: & 0 \\
\hline & Estrategias usadas mf & 4 \\
\hline & Estrategias usadas $\mathrm{f}$ & 0 \\
\hline & Estrategias usadas mfd & 0 \\
\hline & Estrategias usadas bf & 4 \\
\hline & Total estrategias & \\
\hline
\end{tabular}

Fuente: Elaboración propia.

Tabla 7. Frecuencia de uso estrategias. Escala Metacognitivas

\begin{tabular}{|l|l|c|}
\hline \multicolumn{1}{|c|}{$\mathbf{N}^{\circ}$} & \multicolumn{1}{|c|}{ Estrategia } & Frecuencia \\
\hline 24 & Autoconocimiento & $\mathrm{f}$ \\
\hline 25 & Automanejo/ planificación & $\mathrm{f}$ \\
\hline 26 & $\begin{array}{l}\text { Automanejo/ regulación y } \\
\text { evaluación }\end{array}$ & $\mathrm{f}$ \\
\hline & Resumen: & 0 \\
\hline & Estrategias usadas mf & 3 \\
\hline & Estrategias usadas $\mathrm{f}$ & 0 \\
\hline & Estrategias usadas mfd & 0 \\
\hline & Estrategias usadas bf & 3 \\
\hline
\end{tabular}

Fuente: Elaboración propia. 
Tabla 8. Frecuencia de uso estrategias.

Escala de Apoyo

\begin{tabular}{|l|l|c|}
\hline \multicolumn{1}{|c|}{$\mathbf{N}^{\mathbf{1}}$} & \multicolumn{1}{|c|}{ Estrategia } & Frecuencia \\
\hline 27 & Autoinstrucciones & $\mathrm{f}$ \\
\hline 28 & Autocontrol & $\mathrm{mdf}$ \\
\hline 29 & Contradistractoras & $\mathrm{f}$ \\
\hline 30 & Interacciones sociales & $\mathrm{f}$ \\
\hline 31 & Motivación intrínseca y extrínseca & $\mathrm{f}$ \\
\hline 32 & Motivación de escape & $\mathrm{mf}$ \\
\hline & Resumen: & 1 \\
\hline & Estrategias usadas mf & 4 \\
\hline & Estrategias usadas $\mathrm{f}$ & 1 \\
\hline & Estrategias usadas mfd & 0 \\
\hline & Estrategias usadas bf & 6 \\
\hline & Total estrategias & \\
\hline
\end{tabular}

Fuente: Elaboración propia.

Al analizar la tabla 8, Estrategias de Apoyo, las cuales "apoyan", ayudan y potencian el rendimiento de las estrategias de Adquisición, Codificación y las de Recuperación, incrementando la motivación, la autoestima y la atención, en otras palabras, garantizando el buen funcionamiento de todo el sistema cognitivo, tenemos que "Motivación de escape", referida a la habilidad para activar, regular y mantener la conducta de estudio, se detectó en rango "Muy frecuente", es decir, fue muy significativa con más del 75\% de uso; las estrategias de "Autoinstrucciones", "Contradistractoras", "Interacciones sociales" y "Motivación intrínseca y extrínseca", se presentan con una frecuencia de uso de $50 \%$ a $74 \%$, esto es, con uso "Frecuentemente" (f), y por lo tanto son significativas en cuanto a su frecuencia de utilización. Finalmente, la estrategia "Autocontrol", que se relaciona con la capacidad de la persona para controlar estados psicológicos, como por ejemplo la ansiedad, se manifestó a nivel de "Mediana frecuencia" (mdf) y por lo tanto sólo mediana significancia.
A modo de resumen general, de las 32 estrategias ACRA, la única usada en mayor rango de frecuencia, y por lo tanto con mayor relevancia, en este caso a nivel "Muy frecuentemente" (mf) y por consiguiente por sobre un $75 \%$ de utilización por parte de los estudiantes de la muestra, corresponde a la estrategia de "Motivación de escape". Lo anterior corresponde al 3.2\% del porcentaje total de uso de las estrategias de las cinco escalas ACRA. Por otra parte, en rango "Frecuentemente" (f) tenemos 15 estrategias: "Repaso reiterado", "Metáforas", "Aplicaciones", "Secuencias", "Búsqueda de codificaciones", "Búsqueda de indicios", "Planificación de respuestas", "Respuesta escrita", "Automanejo/planificación", "Autoconocimiento", "Automanejo/regulación y evaluación", "Autoinstrucciones", "Contradistractoras", "Interacciones Sociales" у "Motivación intrínseca/extrínseca", lo cual representa el 46.8\% del total de estrategias respectivamente.

En cuanto a las estrategias usadas a nivel "Medianamente frecuente" (mdf), esto es, en un rango de frecuencia entre el $25 \%$ y el $49 \%$, y por tanto 
consideradas como de "Mediana Significancia", tenemos 15 estrategias: "Exploración", "Subrayado lineal", "Subrayado idiosincrático", "Epigrafiado", "Repaso en voz alta", "Repaso mental", "Nemotécnias", "Relaciones intracontenido", "Relaciones compartidas", "Imágenes", "Autopreguntas", "Paráfrasis", "Agrupamientos", "Diagramas" y "Autocontrol", lo cual representa el 46.8\% del total de estrategias.

Finalmente, la única estrategia que aparece en rango "Baja frecuencia", y por tanto con la más baja significancia en cuanto a frecuencia de uso, corresponde a la estrategia "Mapas conceptuales", correspondiente a un $3.2 \%$ de las estrategias.

En general, estos datos muestran una cierta frecuencia de uso de algunas de las estrategias del Modelo ACRA, pero se debe poner también atención al número de estrategias que muestran baja frecuencia de uso, ya que esto implica que son desconocidas para los estudiantes.

\section{Análisis correlacional entre estrategias y rendimiento en inglés}

Para el segundo objetivo específico de la investigación, se correlacionaron los datos de frecuencia de uso de las diferentes estrategias de aprendizaje y el rendimiento de los alumnos de la muestra. Los resultados obtenidos se presentan en la tabla 9.

En función de los datos recogidos, las estrategias que aportan de forma más significativa a la correlación con los resultados académicos, son las siguientes:

- Exploración, estrategia de la escala de Adquisición de información, presentó un $r$ parcial $=0.267$; $r$ cambio $=0.267 \mathrm{y}$ un aporte de $31 \%$.

- Subrayado lineal, también de Adquisición, con un $r$ parcial $=0.337$, un $r$ cambio $=0.07 \mathrm{y}$ un aporte de $8.1 \%$.

- Imágenes, de la escala de Codificación de información: $r$ parcial $=0.537$, un $r$ cambio $=0.052$ y un aporte de $6 \%$.
- Metáforas, también de la escala de Codificación de información, con un $r$ parcial $=0.0631$, un $r$ cambio $=0.094$ y un aporte de $10.9 \%$.

- Autopreguntas, también de la escala de Codificación de la información, con un $r$ parcial $=0.684$, un $r$ cambio $=0.05$ y un aporte de $5.8 \%$.

Considerando que son éstas las estrategias que presentan mayor correlación con los resultados académicos en esta carrera, no se debe olvidar que existen 27 estrategias que no mostraron un grado de correlación significativo. Lo anterior puede deberse a que, como se ha mencionado antes en este estudio, los alumnos no utilizan las estrategias simplemente por desconocimiento. De esta forma, se afianza aún más la necesidad de crear un programa de reforzamiento del uso de estrategias, especialmente las detectadas con baja frecuencia de utilización.

En cuanto a las estrategias que aportan en menor significancia a la correlación con el rendimiento académico de la muestra, y que por lo tanto son menos significativas, tenemos:

- Mapas conceptuales, de la escala de Codificación, con un aporte $=0 \%$.

- Autoconocimiento, automanejo/planificación de la escala Metacognitivas; Contradistractoras, de la escala de Apoyo, y Repaso reiterado, ésta última perteneciente a la escala de Adquisición, con un $0.1 \%$ de aporte a la correlación.

- Repaso mental, de la escala de Adquisición; Paráfrasis, de Codificación; Auto instrucciones y motivación intrínseca y extrínseca, ambas de la escala de Apoyo al procesamiento, las cuales aportan en el rango de $0.2 \%$.

- Aplicaciones, de la escala de Codificación con un $0.3 \%$ de aporte.

- Relaciones compartidas, Búsqueda de indicios y Planificación de respuesta, de la escala de Recuperación, con un $0.5 \%$ de aporte.

El resto de las estrategias se manifestaron en el 
rango de entre $1.2 \%$ a $4.6 \%$ de aporte, pero para efectos de este estudio, interesaba determinar las estrategias con mayor y menor coeficiente de correlación.

\section{Tabla 9. Coeficiente de correlación entre resultados Cuestionario ACRA y rendimiento académico. Asignatura Competencias Comunicativas Integradas en Inglés II}

\begin{tabular}{|c|c|c|c|c|}
\hline$N^{\circ}$ & Estrategia & $r$ parcial & r cambio & $\%$ aporte \\
\hline 1 & Exploración & 0.267 & 0.267 & 31.0 \\
\hline 2 & Subrayado lineal & 0.337 & 0.07 & 8.1 \\
\hline 3 & Subrayado idiosincrático & 0.377 & 0.04 & 4.6 \\
\hline 4 & Epigrafiado & 0.405 & 0.028 & 3.3 \\
\hline 5 & Repaso voz alta & 0.439 & 0.034 & 3.9 \\
\hline 6 & Repaso mental & 0.441 & 0.002 & 0.2 \\
\hline 7 & Repaso reiterado & 0.442 & 0.001 & 0.1 \\
\hline 8 & Nemotecnias & 0.456 & 0.014 & 1.6 \\
\hline 9 & Relaciones intracontenido & 0.481 & 0.025 & 2.9 \\
\hline 10 & Relaciones compartidas & 0.485 & 0.004 & 0.5 \\
\hline 11 & Imágenes & 0.537 & 0.052 & 6.0 \\
\hline 12 & Metáforas & 0.631 & 0.094 & 10.9 \\
\hline 13 & Aplicaciones & 0.634 & 0.003 & 0.3 \\
\hline 14 & Autopreguntas & 0.684 & 0.05 & 5.8 \\
\hline 15 & Paráfrasis & 0.686 & 0.002 & 0.2 \\
\hline 16 & Agrupamientos & 0.709 & 0.023 & 2.7 \\
\hline 17 & Secuencias & 0.741 & 0.032 & 3.7 \\
\hline 18 & Mapas conceptuales & 0.741 & 0 & 0.0 \\
\hline 19 & Diagramas & 0.763 & 0.022 & 2.6 \\
\hline 20 & Búsqueda de codificaciones & 0.775 & 0.012 & 1.4 \\
\hline 21 & Búsqueda de indicios & 0.779 & 0.004 & 0.5 \\
\hline 22 & Planificación de respuesta & 0.783 & 0.004 & 0.5 \\
\hline 23 & Respuesta escrita & 0.793 & 0.01 & 1.2 \\
\hline 24 & Autoconocimiento & 0.794 & 0.001 & 0.1 \\
\hline 25 & Automanejo/planificación & 0,795 & 0.001 & 0.1 \\
\hline 26 & Automanejo/regulación y evaluación & 0.805 & 0.01 & 1.2 \\
\hline 27 & Auto instrucciones & 0.807 & 0.002 & 0.2 \\
\hline 28 & Autocontrol & 0.827 & 0.02 & 2.3 \\
\hline 29 & Contradistractoras & 0.828 & 0.001 & 0.1 \\
\hline 30 & Interacciones sociales & 0.839 & 0.011 & 1.3 \\
\hline 31 & Motivación intrínseca /extrínseca & 0.841 & 0.002 & 0.2 \\
\hline \multirow[t]{2}{*}{32} & Motivación de escape & 0.861 & 0.02 & 2.3 \\
\hline & & & 0.861 & 100.0 \\
\hline
\end{tabular}

Fuente: Elaboración propia. 


\section{Discusión}

En cuanto al estudio sistemático de estrategias de aprendizaje y su relación con la adquisición de una lengua extranjera, en términos globales se podría comentar que es un tema de importancia en países de habla inglesa; no así en Latinoamérica, como es el caso puntual de Chile. Así, es posible pensar en la necesidad de realizar estudios más profundos y sistemáticos en la temática de las estrategias de aprendizaje y la adquisición de un idioma extranjero, ya que existe una supuesta ausencia de bases teóricas en las cuales sustentar la investigación en esta área, hecho que es necesario comprobar y analizar en detalle a través de una revisión crítica y exhaustiva de toda la investigación existente en la especialidad, no realizada hasta la fecha. Del mismo modo, existen problemas teóricos y metodológicos que dificultan poder establecer la verdadera relación entre uso de estrategias y aprendizaje de lenguas. Especial importancia adquiere el análisis de la operacionalización del constructo "aprendizaje" y "éxito" de los programas de entrenamiento estratégico, tarea que tampoco ha sido abordada.

Además, las implicaciones teóricas y aplicadas de la investigación sobre estrategias estarían condicionadas por los hallazgos de la investigación existente y futura sobre dos hechos: el carácter lineal o no entre nivel de dominio de la segunda lengua, o lengua extranjera, y uso de estrategias, y la relación entre estrategias y aprendizaje, en el sentido de poder aclarar si las estrategias son una ayuda al aprendizaje o el resultado de dicho aprendizaje.

Por otra parte, no existen trabajos que hayan analizado exhaustivamente estos dos aspectos en la investigación existente o disponible en este campo específico, lo cual invita a establecer desafíos en este ámbito, a través de investigación organizada que promueva el avance científico en el tema. Finalmente y de acuerdo con la bibliografía consultada en este estudio, existen trabajos realizados en este campo, pero éstos no analizan críticamente las bases psicológicas, lingüísticas y educativas sobre las que se sustentan las propuestas sobre programas de entrenamiento estratégico para la adquisición de idiomas extranjeros. Estos conceptos se sustentan tanto en la propia experiencia de este investigador, como en los trabajos de algunos autores destacados en el tema. De esta forma, los problemas teóricos y metodológicos en este campo de investigación han sido comentados en los trabajos de Dörnyei et al., (2003), Manchón (1997b, 2001) y McDonough (1995), entre otros. Todos ellos dan especial relevancia a la necesidad de plantear la investigación futura con una base teórica más sólida, considerando la poca utilidad del concepto de estrategia en su aplicación al aprendizaje de lenguas, debido a la falta de precisión en su definición operacional, la cual ha guiado la investigación existente, e involucrando los avances en psicología sobre el mismo tema, es decir, trabajo interdisciplinar.

Manchón (2005) argumenta la necesidad de analizar críticamente los datos empíricos disponibles sobre la relación entre uso de estrategias y aprendizaje, y la conveniencia de la inclusión de programas de entrenamiento estratégico en la enseñanza de segundas lenguas. En el mismo ámbito, Dörnyei et al. (2003) abogan por la necesidad de establecer una agenda de investigación futura que tenga en cuenta teorías psico-cognitivas del aprendizaje, en general, y del aprendizaje lingüístico, en particular, así como la investigación sobre auto-regulación en procesos de aprendizaje.

En consecuencia y luego de la revisión bibliográfica, el autor coincide plenamente con importantes investigadores que han profundizado en la temática del uso de estrategias de aprendizaje como medio para facilitar la adquisición de un idioma extranjero ya que, considerándolos antecedentes en lo que respecta al modo en que se ha definido operacionalmente la idea de "estrategia", las estrategias objeto de análisis en cada trabajo, y sus planteamientos metodológicos, se hace pertinente una revisión 
exhaustiva de los mismos, la cual permita discernir con claridad la verdadera naturaleza de la relación entre uso de estrategias y avance en el aprendizaje, sobre todo ahora que el inglés es considerado la lingua franca. De esta forma, tal como comenta McDonough (1995) en su trabajo de revisión sobre este tema, es de vital importancia poder dilucidar si las estrategias, como se mencionó anteriormente, contribuyen al aprendizaje o son el resultado de éste.

De esta forma, existe una clara necesidad de establecer procedimientos sistemáticos respecto de la instalación de programas de entrenamiento de estrategias como apoyo al proceso enseñanza-aprendizaje de idiomas extranjeros, lo cual es coincidente con la opinión de otros autores como Chamot et al. (1994a) y Wenden (1991).

No obstante los planteamientos expuestos anteriormente, es interesante comentar que en la realidad chilena, aunque los tres últimos gobiernos han favorecido la instalación del idioma inglés como herramienta de desarrollo para el país, iniciándose su enseñanza desde nivel preescolar en los colegios particulares pagados, y desde el quinto año de enseñanza básica en los establecimientos municipalizados en forma obligatoria, no se advierten avances significativos en la adquisición de la lengua.

Más aún, la investigación en el campo de Learning Acquisition en Chile, en cuanto al apoyo de las estrategias de aprendizaje, es extremadamente escasa, mientras que en otros países ha sido relativamente constante desde los años ochenta; por lo anterior, es evidente la falta de un mayor debate en este campo y sobre las consecuencias que este hecho tiene a la hora de valorar la fundamentación y resultados en otras investigaciones similares. Tal como se menciona en el marco teórico de este estudio, y consultadas varias fuentes de información, no hay hasta ahora una revisión crítica acerca de la investigación existente. En el caso específico del aprendizaje de idiomas, como el inglés, al menos en Chile no existe registro, y de ahí el carácter exploratorio de este trabajo. Por lo anterior, esta temática se convierte en un campo de investigación altamente fértil para el desarrollo de investigaciones posteriores que permitan elaborar algún tipo de batería o programa de entrenamiento para apoyar las estrategias que son utilizadas y fortalecer el uso de las que no lo son. Por otro lado, respecto de estudios realizados en el campo de la relación entre uso de estrategias de aprendizaje y el rendimiento académico, la investigación realizada por Valle et al. (1998: 27-53) y otros, confirman que los estudiantes que utilizan un número mayor de estrategias de aprendizaje logran un mejor rendimiento académico (Camarero et al., 2000: 615622; Cano et al., 1991: 69-86).

Extrapolando los resultados al sistema de formación tradicional en Chile, podría decirse que los resultados pueden interpretarse desde los códigos institucionales en tradiciones educativas fuertemente enmarcadas en el control del comportamiento.

También puede vislumbrarse como un indicador de la tendencia de los estudiantes a privilegiar aprendizajes superficiales, escasamente significativos y que no requieren pensamiento autónomo, en detrimento de procesos de selección, organización y elaboración de información. Las estrategias de aprendizaje son especialmente importantes para el aprendizaje puesto que constituyen herramientas para el desarrollo de competencias comunicativas básicas; en tal sentido, estos resultados podrían ser usados para el planteamiento de un eventual plan de intervención tendiente a desarrollar habilidades cognitivas y metacognitivas en el alumno. Es así que se concuerda con Monereo (1997), en cuanto a que para conseguir alumnos estratégicos es importante contar con profesores estratégicos, que hayan tomado conciencia de los complejos procesos cognitivos y metacognitivos que requieren sus alumnos para aprender.

Por otra parte, los resultados de la presente investigación coinciden con los obtenidos por otros investigadores que han hecho uso de diferentes constructos, 
tales como los enfoques y los estilos de aprendizaje (Abalde et al., 2001: 465-489; Hernández et al., 2001: 303-318; 2002: 487-510; 2005: 283-299; 2000; Valle et al., 1998: 393-412; 2000: 368-375), todos ellos relacionados con enfoques de enseñanza y rendimiento, en los cuales se establece que la frecuencia de uso de estrategias de aprendizaje es muy poco significativa. En la misma línea, al igual que el estudio de Printich et al. (1991), esta investigación identificó correlaciones significativas entre estrategias de aprendizaje y rendimiento académico. Coincide también con el estudio de Roces et al. (1999: 41-50), en el cual se determinaron correlaciones positivas y significativas entre la mayoría de los factores de estrategias de aprendizaje evaluados, en su caso, por el Cuestionario de Estrategias de aprendizaje y motivación (CEAM) II y el rendimiento académico.

Del mismo modo, de acuerdo con Marugán (2008):

Las estrategias de aprendizaje se constituyen en una importante variable moduladora del rendimiento en comprensión y memoria, en la medida en que son los alumnos más estratégicos los que obtienen puntuaciones superiores en la prueba, especialmente en la subprueba de memoria. Así mismo, a tenor del análisis efectuado con la variable dependiente "rendimiento académico", se puede afirmar que las estrategias de aprendizaje sí parecen tener un peso específico en su estudio, ya que existe una clara tendencia a obtener mejores calificaciones en las diferentes evaluaciones por aquellos alumnos que también puntúan alto en estrategias y viceversa.

Como sustento de los resultados en cuanto al tema del rendimiento académico, se han considerado además los trabajos de Andrade et al. (2000), donde se establece que la calificación es la apreciación del rendimiento académico, y los estudios de Reyes (2004), los cuales plantean que el bajo rendimiento académico de los estudiantes es resultado de diversas razones, como la deficiencia en el uso de estrategias de aprendizaje. Esta relación se sustenta en los trabajos de Beltrán (1998), quien sostiene que las estrategias de aprendizaje sirven para mejorar la calidad del rendimiento académico. Así mismo, De Zubiría et al. (1996) afirman que las estrategias de aprendizaje desarrollan el pensamiento que exige la puesta en marcha de su capacidad de pensar. Gargallo et al. (2000) concluyen en su investigación que la calificación ha mejorado con el uso del mayor número de estrategias de aprendizaje, mismas que deben incluirse en el proyecto curricular, tanto de los establecimientos de educación básica como secundaria y en las universidades.

La investigación de Bara (2001) afirma que el uso de las estrategias de aprendizaje favorece la construcción de aprendizajes, ya que es ahí donde el objetivo de la educación adquiere significado. Por su parte, Hernández (2005) afirma que el uso de las estrategias de aprendizaje en el proceso de enseñanza-aprendizaje crea las condiciones pedagógicas y psicológicas para alcanzar mayor grado de conciencia e independencia en el proceso de asimilación.

Así, se puede señalar finalmente que el uso de estrategias de aprendizaje se relaciona significativamente con el rendimiento académico, pero es preciso velar por una utilización adecuada y sistemática de éstas. En consecuencia, las estrategias que muestran bajo nivel de correlación con los resultados académicos deben ser reforzadas en cuanto a su uso, para permitir a los estudiantes obtener un rango mayor de posibilidades para mejorar su desempeño académico.

En la realidad globalizada en que nos encontramos inmersos, este planteamiento es totalmente coherente con la filosofía misma, que reclama una enseñanza centrada en el aprendizaje del estudiante, al cual debemos dotar de herramientas cognitivas que le ayuden y permitan desenvolverse de modo autónomo en la sociedad de la información y el conocimiento. 
Esta tarea puede efectuarse mediante alguna asignatura específica o taller de tipo instrumental y transversal en los cursos iniciales de cada programa de estudio. También puede ser implementada mediante la introducción, en nuestra docencia diaria, de procesos reflexivos y dialógicos con los estudiantes sobre cómo aprender los contenidos, y acerca de la práctica necesaria para el logro de estas competencias. Este segundo planteamiento, más interesante a nivel teórico, pues asume la integración de las estrategias de aprendizaje en las propias materias o cursos que imparten los distintos profesores, e incluso a nivel pragmático porque evita las suspicacias y posiciones defensivas a que puede llevar la primera opción antes mencionada, pasa por un modelo de profesor "centrado en el aprendizaje", que diseña su curso en torno de un aprendizaje en que se favorezca la construcción de conocimiento entre todos, con la propia ayuda y mediación. Un profesor centrado en el aprendizaje no es más que un educador consciente y responsable que llega también a ser un profesor estratégico que con el paso del tiempo enseñe a sus alumnos a ser estratégicos también.

Hablamos de un profesor que debe "saber del aprendizaje de su materia", no sólo de su enseñanza, un profesor que debe conocer las dificultades de ese aprendizaje, así como los procedimientos para aprenderla mejor, y por supuesto, altamente consciente de los objetivos que espera lograr y de los criterios e instrumentos que utilizará. Trabajando de esta forma, un profesor centrado en el aprendizaje ha de incluir en su docencia periodos en que se reflexione sobre estos temas con sus alumnos. Un profesor de este estilo ha de actuar como un mediador capaz de organizar el trabajo en el aula para crear entornos de aprendizaje efectivo, donde se trabaje para resolver problemas reales, con tareas auténticas, para que los alumnos indaguen, cuestionen, reconstruyan, cooperen con los pares, etcétera. Es éste el punto en donde concurren la metodología docente coherente con tales planteamientos y ajustada a objetivos de excelencia, que van mucho más lejos que repetir lo aprendido: metodología expositiva cuando sea necesario, estudio de casos, resolución de problemas, simulaciones, trabajos de investigación; y una evaluación formativa y continua, insertada en el proceso de enseñanza-aprendizaje, y aportando a los estudiantes diferentes claves, indicios y recomendaciones para la mejora, de manera tal que el alumno interiorice criterios de calidad que le permitan aprender a autoevaluarse y a mejorar el propio proceso de aprendizaje, todo lo cual nos lleva a promover una oferta racional de formación de profesores, ya sea en ejercicio en establecimientos de educación básica, media o universitaria, adaptado a las necesidades que generan las nuevas políticas de convergencia, las cuales requieren de profesores centrados en el aprendizaje, capaces de estimular el desarrollo personal y autónomo de los estudiantes.

Finalmente, en el contexto universitario chileno, casi la totalidad de las carreras de los diferentes planteles de educación superior cuentan con uno o al menos dos semestres de inglés técnico, excepto las pedagogías en inglés. Los cursos de estos periodos están enfocados al desarrollo idiomático comunicacional básico y/o al uso de estrategias de lectura comprensiva de textos técnicos escritos en inglés acorde con cada especialidad. En este sentido, la creación de un programa de mejora para la adquisición de esta lengua, a través del apoyo mediante el desarrollo de estrategias de aprendizaje como las identificadas por las escalas ACRA, sería altamente beneficiosa para esta población educacional universitaria, a través de la implementación de talleres de reforzamiento de estrategias de aprendizaje, como las trabajadas en este estudio. 


\section{Referencias}

Abalde, E., M. Muñoz, L. Buendía, E. Olmedo, E. Berrocal, J. Cajide, J. Soriano, F. Hernández Pina, M. P. García y J. Maquillón (2001), "Los enfoques de aprendizaje en estudiantes universitarios españoles", en Revista de Investigación Educativa, 19 (2), pp. 465-489.

Andrade, G. M., J. C. Miranda y S. I. Freixas (2000), Influencias del rendimiento académico, Lima, UNMSM.

Bara, P. M. (2001), "Estrategias metacognitivas y de aprendizaje: estudio empírico sobre el efecto de la aplicación de un programa metacognitivo, y el dominio de las estrategias del aprendizaje en estudiantes de ESO, BUP", Tesis doctoral, Universidad Complutense de Madrid, <http://www.ucm.es/eprints/4765/13k> [Consulta: abril 2014].

Beltrán, J. A. (1993), Procesos, estrategias y técnicas de aprendizaje, Valle Hermoso, Madrid, Síntesis.

Cano, F. y F. Justicia (1991), "Estrategias de aprendizaje, especialidades y rendimiento académico", en Revista de Psicología de la Educación, vol. 3, núm. 1, pp. 69-86.

Camarero, F., F. Martín y J. Herrero (2000), "Estilos y estrategiasde aprendizaje en estudiantes universitarios", en Psicothema, 12 (4), pp. 615-622.

Chamot, A. U. y J. M. O'Malley (1994a), "Instructional approaches and teaching procedures", en K. S. Urbschat y R. Pritchard (eds.), Kids come in all languages: reading instruction for ESL students, Newark, International Reading Association.

De Zubiría, M. y J. de Zubiría (1996), Biografia del pensamiento, estrategias para el desarrollo de la inteligencia, Colombia, Cooperativa Editorial Magisterio.

Dörnyei, Z. y P. Skehan (2003), "Individual differences in second language learning", en C.J. Doughty y M. H. Long (eds.), The Handbook of Second Language Acquisition, Oxford, Blackwell Publishing Ltd.

Ellis, G. F. y B. Sinclair (1989), Learning to learn english learner's book: a course in learner training, Cambridge, U. Press.

Gargallo, B. y A. Ferreras (2000), Estrategias de aprendizaje. Un programa de intervención para ESO y EPA, Madrid, Fureso.
Hernández, A. (2005), "Las estrategias de aprendizaje como un medio de apoyo en el proceso de asimilación", Tesis, La Habana, <http://www.cepes.uh.cu/ bibliomaestria/estrategias como medio.doc $>$ [Consulta: diciembre 2014].

Hernández, F. y J. J. Maquillón (2000), “Enfoques de aprendizaje en alumnos de COU y reforma que pretenden acceder a la universidad y alumnos de primer curso de carrera", en Varios, Congrésd'Orientación Universitaria, Barcelona, Ediciones de la Universitat de Barcelona.

Hernández, F., M. P. García y J. J. Maquillón (2001), "Estudio empírico de los enfoques de aprendizaje de los estudiantes universitarios en función del perfil de su titulación (profundo v/s vertical)", en Revista Española de Orientación y Psicopedagogía, 12 (22), pp. 303- 318.

Hernández, F., M. P. García, P. Martínez, R. M. Hervás y J. J. Maquillón (2002), "Consistencia entre motivos y estrategias de aprendizaje en estudiantes universitarios", en Revista de Investigación Educativa, 20 (2), pp. 487-510.

Hernández, F. y R. Hervás (2005), "Enfoques y estilos de aprendizaje en educación superior", en Revista Española de Educación y Psicopedagogía, 16 (2), pp. 283-299.

Hsiao, T. Y. y R. L. Oxford (2002), "Comparing theories of language learning strategies: a confirmatory factor analysis", en The Modern Language fournal, 86, pp. 368-383.

Manchón, R. M., L. Murphy y J. Roca de Larios (1997), "La investigación sobre escritura en lengua extranjera: planteamientos teóricos e implicaciones pedagógicas", ponencia presentada en el XV Congreso de la Asociación Española de Lingüistica Aplicada, Zaragoza, abril de 1997.

Manchón, R. M (1998), "Broadening the concept of communication strategies in interlanguage communication", en E. Alcón Soler y V. Codinapur (eds.), Current trends in English language methodology, Castelló, Servei de Publicacions-Universitat Jaume I, pp. 101-118.

Manchón, R. M. (2001), “Trends in the conceptualization of second language composing strategies: a critical analysis", en R. M. Manchón (ed.), Writing in the L2 classroom: issues in research and pedagogy, International fournal of English Studies, 1 (2), pp. 27-70. 
Manchón, R. M. (2005), "Estrategias de aprendizaje y segundas lenguas", en Convocatoria de ayudas de Proyectos de Investigación [Memoria científico-técnica del Proyecto], Newcastle, CambridgeScholars Publishing.

Marugán, M. (2008), "Importancia de las estrategias generales de aprendizaje en el rendimiento escolar", en Quaderns Digitals, núm. 55.

McDonough, S. H. (1995), Learner strategies, Essex, University of Essex.

Mendelsohn, D. (1994), Learning to listen: a strategy based approach for the second language learner, San Diego, Dominie Press.

Monereo, C. (1997), "La construcción del conocimiento estratégico en el aula”, en M. L. Pérez Cabaní, La enseñanza y el aprendizaje de estrategias desde el currículum, Gerona, Horsi.

O’Malley, F. M. y A. C. Chamot (1990), Learning strategies in second language acquisition, EUA, C.U.P.

Oxford, R. y A. D. Cohen (1992), "Language learning strategies: crucial issues of concept and classification", en Applied Language Learning, vol. 3, núms. 1-2, pp. 1-35.

Pintrich, P. R. y T. García (1991), "Student goal orientation and self regulation in the class-room", en M. L. Maher y P. R. Printich (eds.), Advances in motivation and achievement (vol. 7), Greenwich, CT, JAI Press.

Rees-Miller, J. (1990), "A critical appraisal of learner training: theoretical bases and teaching implications", en TESOL Quarterly, vol. 28, núm. 4, pp. 776 -781.

Reyes, R. y L. Salvador (2004), "Rendimiento académico de los estudiantes universitarios", en Revista Theorethikos, San Salvador, Universidad San Francisco Gavidia.

Ridgway, T. (2000), Listening strategies-I beg your pardon?, Oxford, Oxford University Press.

Roces, C., J. A. González-Pienda, J. C. Nuñez, S. González-Pumariega, M. S. García y L. Álvarez (1999), "Relaciones entre motivación, estrategias de aprendizaje y rendimiento académico en estudiantes universitarios. Mente y conducta en situación Educativa", en Revista electrónica del Departamento de Psicología, Universidad de Valladolid, 1 (I), pp. 41-50.

Román, J. M. y S. Gallego (1994), ACRA: Escalas de Estrategias de Aprendizaje, Madrid, TEA Ediciones SA. [Manual, Cuadernillo y Hoja de Respuestas] [8 ${ }^{\mathrm{a}}$ ed., marzo 2008].

Román, J. M. y L. Poggioli (2013), ACRA (r): Escalas de estrategias de aprendizaje, Caracas, Publicaciones UCAB (Postgrado Doctorado en Educación).

Rubin, J. e I. Thompson (1994), How to be a more successful language learner, Boston, Heinle and Heinle Publishers.

Valle, A. y A. Rodríguez (1998), "Estrategias de aprendizaje y rendimiento académico", en Boletín de Psicología, 60, pp. 27-53.

Valle, A., R. González Cabanach, J. Núñez y J. A. González-Pienda (1998), "Variables cognitivomotivacionales, enfoques de aprendizaje y rendimiento académico", en Psicothema, 10 (2), pp. 393-412.

Valle, A., R. González Cabanach, J. Núñez, J. M. Suárez, I. Piñeiro y S. Rodríguez, (2000), "Enfoques de aprendizaje en estudiantes universitarios", en Psicothema, 12 (3), pp. 368-375.

Vidal-Abarca, E. (1991), Comprender para aprender. Un programa para mejorar la comprensión y el aprendizaje de textos, Madrid, CEPE.

Weaver, S. J. y A. D. Cohen (1997), Strategies-based instruction: A teacher-training manual, Minneapolis, Center for Advanced Research on Language Acquisition, University of Minnesota.

Wenden, A. (1991), Learner Strategies for Learner Autonomy, Nueva York, Prentice Hall.

Willing, K. (1989), Teaching how to learn: learning strategies in ESL, Sydney, National Centre for English Language Teaching and Research, Macquarie University.

\section{Cómo citar este artículo:}

Cárdenas-Narváez, Juan-Carlos (2019), "Relación entre estrategias de aprendizaje y rendimiento académico en alumnos de pedagogía en inglés", en Revista Iberoamericana de Educación Superior (RIES), México, UNAM-IISUE/Universia, vol. X, Núm. 27, pp. 115-135, DOI: dx.doi.org/10.22201/ iisue.20072872e.2019.27.343 [consulta: fecha de última consulta]. 